\title{
Appendix 3 Seychelles Case Study
}

Table A3.1 Visitor arrivals

to Seychelles (1970-2011)

\begin{tabular}{|c|c|}
\hline Year & $\begin{array}{c}\text { Number of } \\
\text { visitor arrivals }\end{array}$ \\
\hline 1970 & 1,622 \\
\hline 1971 & 3,175 \\
\hline 1972 & 15,197 \\
\hline 1973 & 19,464 \\
\hline 1974 & 25,932 \\
\hline 1975 & 37,321 \\
\hline 1976 & 49,498 \\
\hline 1977 & 54,490 \\
\hline 1978 & 64,995 \\
\hline 1979 & 78,852 \\
\hline 1980 & 71,762 \\
\hline 1981 & 60,425 \\
\hline 1982 & 47,280 \\
\hline 1983 & 55,867 \\
\hline 1984 & 63,417 \\
\hline 1985 & 72,542 \\
\hline 1986 & 66,782 \\
\hline 1987 & 71,626 \\
\hline 1988 & 77,401 \\
\hline 1989 & 86,093 \\
\hline 1990 & 103,770 \\
\hline 1991 & 90,050 \\
\hline 1992 & 98,547 \\
\hline 1993 & 116,181 \\
\hline 1994 & 109,901 \\
\hline 1995 & 102,716 \\
\hline 1996 & 130,995 \\
\hline 1997 & 130,070 \\
\hline 1998 & 128,258 \\
\hline 1999 & 124,865 \\
\hline 2000 & 130,046 \\
\hline 2001 & 129,762 \\
\hline 2002 & 132,246 \\
\hline 2003 & 122,038 \\
\hline 2004 & 120,765 \\
\hline 2005 & 128,654 \\
\hline 2006 & 140,627 \\
\hline 2007 & 161,273 \\
\hline 2008 & 158,952 \\
\hline 2009 & 157,541 \\
\hline 2010 & 174,529 \\
\hline 2011 & 194,476 \\
\hline
\end{tabular}


Table A3.2 Direct contribution of travel and tourism to GDP (\%), 2004-10

\begin{tabular}{llllllll}
\hline & 2004 & 2005 & 2006 & 2007 & 2008 & 2009 & 2010 \\
\hline Contribution to GDP (\%) & 17.7 & 19.2 & 21.5 & 25.9 & 26.5 & 28.5 & 27.5 \\
\hline
\end{tabular}

Source: Seychelles Tourism Master Plan 2012-20: 17

Table A3.3 Direct and indirect tourism contribution to current GDP by industry, 2007-10

\begin{tabular}{|c|c|c|c|c|c|c|c|c|}
\hline \multirow[b]{2}{*}{ Industry } & \multicolumn{4}{|c|}{ Current SCR m (US\$) ${ }^{a}$} & \multicolumn{4}{|c|}{ In $\%$ total value-added } \\
\hline & 2007 & 2008 & 2009 & 2010 & 2007 & 2008 & $\begin{array}{l}2009 \\
\text { (prov.) }\end{array}$ & $\begin{array}{c}2010 \\
\text { (prov.) }\end{array}$ \\
\hline $\begin{array}{l}\text { Wholesale and retail, } \\
\text { repair motor vehicles }\end{array}$ & 41.8 & 61.0 & 80.1 & 74.3 & 2.3 & 2.5 & 2.5 & 2.3 \\
\hline Transportation & 469.0 & 516.7 & 626.4 & 501.8 & 26.0 & 21.4 & 19.2 & 15.7 \\
\hline $\begin{array}{l}\text { Accommodation and } \\
\text { food service activities }\end{array}$ & 994.4 & $1,492.6$ & $1,944.3$ & $1,984.0$ & 55.1 & 61.8 & 59.6 & 62.0 \\
\hline $\begin{array}{l}\text { Administrative and } \\
\text { support service } \\
\text { activities }\end{array}$ & 114.7 & 128.3 & 202.0 & 223.0 & 6.4 & 5.3 & 6.2 & 7.0 \\
\hline $\begin{array}{l}\text { Arts, entertainment } \\
\text { and recreation }\end{array}$ & 4.7 & 5.6 & 6.5 & 6.7 & 0.3 & 0.2 & 0.2 & 0.2 \\
\hline Taxes on products & 178.8 & 210.0 & 403.9 & 411.8 & 9.9 & 8.7 & 12.4 & 12.9 \\
\hline Total tourism & $1,803.3$ & $2,414.3$ & $3,263.2$ & $3,201.6$ & 100 & 100 & 100 & 100 \\
\hline $\begin{array}{l}\text { Total GDP at current } \\
\text { market prices }\end{array}$ & $6,962.5$ & $9,100.8$ & $11,450.4$ & $11,612.3$ & - & - & - & - \\
\hline $\begin{array}{l}\text { Tourism related } \\
\text { contribution (\%) }\end{array}$ & 25.9 & 26.5 & 28.5 & 27.5 & - & - & - & - \\
\hline
\end{tabular}

${ }^{a}$ Exchange rate taken from xe.com. US $\$ 1=$ SCR14.52

Source: Seychelles Tourism Master Plan 2012-20: 18 
Table A3.4 Visitor expenditure on travel and tourism services and products, 2001-2007 ( $\%$ of total visitor expenditure)

\begin{tabular}{lcrrrrrr}
\hline & $\mathbf{2 0 0 1}$ & $\mathbf{2 0 0 2}$ & $\mathbf{2 0 0 3}$ & $\mathbf{2 0 0 4}$ & $\mathbf{2 0 0 5}$ & $\mathbf{2 0 0 6}$ & $\mathbf{2 0 0 7}$ \\
\hline Large hotels & 51 & 50 & 51 & 51 & 55 & 55 & 55 \\
Small hotels and guesthouses & 13 & 13 & 12 & 10 & 9 & 8 & 5 \\
Of which is from residents & 3 & 3 & 3 & 3 & 3 & 3 & 3 \\
Total accommodation & $\mathbf{6 3}$ & $\mathbf{6 3}$ & $\mathbf{6 3}$ & $\mathbf{6 1}$ & $\mathbf{6 4}$ & $\mathbf{6 3}$ & $\mathbf{6 1}$ \\
Restaurants & 12 & 12 & 11 & 11 & 10 & 10 & 11 \\
Car hire & 4 & 4 & 4 & 3 & 3 & 3 & 2 \\
Prepayments for car hire and & 2 & 2 & 2 & 2 & 2 & 2 & 2 \\
excursions & & & & & & & \\
Taxis and buses & 2 & 2 & 1 & 2 & 1 & 1 & 1 \\
Excursions & 10 & 11 & 11 & 11 & 10 & 10 & 9 \\
Handicrafts & 4 & 5 & 4 & 5 & 4 & 4 & 4 \\
Other Shopping & 0.3 & 1 & 2 & 2 & 2 & 1 & 1 \\
Unallocated & 4 & 2 & 1 & 2 & 2 & 2 & 2 \\
Cruise passenger and short-stay $_{\text {transit passengers }}^{\text {b }}$ & 1 & 0.4 & 1 & 1 & 1 & 1 & 1 \\
\hline
\end{tabular}

aPrepayments estimated and based on expenditure outside the hotel. bEstimate only.

Source: McEwen and Bennett (2010)

Table A3.5 Visitor expenditure 2007-10: total spend, spend per visitor and daily spend per visitor

\begin{tabular}{|c|c|c|c|c|c|c|}
\hline & \multicolumn{2}{|c|}{ Tourism spend } & \multicolumn{2}{|c|}{ Spend per visitor } & \multicolumn{2}{|c|}{ Daily spend per visitor } \\
\hline & $€ \mathrm{~m}^{\mathrm{a}}$ (US\$m) & $\%$ Change & $€ \mathrm{~m}(\mathrm{US} \$ \mathrm{~m})$ & $\%$ Change & $€ \mathrm{~m}$ (US\$m) & $\%$ Change \\
\hline 2007 & $\begin{array}{c}219.3 \\
(269.3)\end{array}$ & - & $\begin{array}{c}1,359.6 \\
(1,670.2)\end{array}$ & - & $\begin{array}{c}137.3 \\
(168.6)\end{array}$ & - \\
\hline 2008 & $\begin{array}{c}221.5 \\
(272.1)\end{array}$ & 1.0 & $\begin{array}{c}1,393.7 \\
(1,712.2)\end{array}$ & 2.5 & $\begin{array}{c}138.0 \\
(169.5)\end{array}$ & 0.5 \\
\hline 2009 & $\begin{array}{c}201.1 \\
(247.1)\end{array}$ & -9.2 & $\begin{array}{c}1,276.3 \\
(1,567.9)\end{array}$ & -8.4 & $\begin{array}{c}125.1 \\
(308.4)\end{array}$ & -9.3 \\
\hline 2010 & $\begin{array}{l}232.8 \\
(286)\end{array}$ & 15.8 & $\begin{array}{c}1,334.0 \\
(1,638.8)\end{array}$ & 4.5 & $\begin{array}{c}128.3 \\
(157.6)\end{array}$ & 2.5 \\
\hline
\end{tabular}

aExchange rate taken from xe.com. 1US\$ $=€ 0.814$

Source: Seychelles Tourism Master Plan 2012-20: 21 
Table A3.6 Direct contribution to GST (\%): tourism sector activities, 2007-10

\begin{tabular}{lcccc}
\hline & \multicolumn{4}{c}{ Contribution to tourism GST receipts (\%) } \\
\cline { 2 - 5 } & $\mathbf{2 0 0 7}$ & $\mathbf{2 0 0 8}$ & $\mathbf{2 0 0 9}^{\mathrm{a}}$ & $\mathbf{2 0 1 0}^{\mathrm{b}}$ \\
\hline Accommodation & $\mathbf{8 4 . 3}$ & $\mathbf{8 3 . 8}$ & $\mathbf{8 3 . 2}$ & $\mathbf{8 2 . 9}$ \\
Five star hotels & 47.8 & 47.9 & 46.7 & 49.3 \\
Large hotels & 30.5 & 28.8 & 27.6 & 23.2 \\
Guest houses and self-catering & 6.0 & 7.0 & 9.0 & 10.3 \\
Other land-based tourism activities & $\mathbf{6 . 3}$ & $\mathbf{5 . 5}$ & $\mathbf{5 . 2}$ & $\mathbf{3 . 9}$ \\
Marine-based tourism activities & $\mathbf{3 . 5}$ & $\mathbf{5 . 3}$ & $\mathbf{5 . 9}$ & $\mathbf{7 . 3}$ \\
Transport-based activities & $\mathbf{5 . 9}$ & $\mathbf{5 . 4}$ & $\mathbf{5 . 6}$ & $\mathbf{5 . 9}$ \\
\hline
\end{tabular}

aln 2009, total GST receipts from the tourism sector: SCR372 m: 23

bIn 2010, total GST receipts from the tourism sector: SCR376.8 m: 23

Source: Seychelles Tourism Master Plan 2012-20: 24

Table A3.7 Visitor average length of stay 2000-11

\begin{tabular}{lcccccccccccccc}
\hline & 2000 & 2001 & 2002 & 2003 & 2004 & 2005 & 2006 & 2007 & 2008 & 2009 & 2010 & $\begin{array}{c}2011 \\
\text { (estimated) }\end{array}$ \\
\hline $\begin{array}{l}\text { Number } \\
\text { of days }\end{array}$ & 10.4 & 10.4 & 10.1 & 10.1 & 10.0 & 9.7 & 9.8 & 9.9 & 10.1 & 10.2 & 10.4 & 10.2 \\
\hline
\end{tabular}

Source: Seychelles Tourism Master Plan 2012-20: 30

Table A3.8 Number of cruise ship arrivals per year, 2004-11

\begin{tabular}{lc}
\hline & Number of ships \\
\hline 2004 & 60 \\
2005 & 42 \\
2006 & 40 \\
2007 & 38 \\
2008 & 34 \\
2009 & 39 \\
2010 & 20 \\
2011 (Jan-Oct only) & 11 \\
\hline
\end{tabular}

Source: Seychelles Tourism Master Plan 2012-20: 32 
Table A3.9 Weekly flights by airline, 2005-2010a

\begin{tabular}{lrrrrrr}
\hline & \multicolumn{7}{c}{ Number of weekly flights (international) } \\
\cline { 2 - 6 } & $\mathbf{2 0 0 5}$ & $\mathbf{2 0 0 6}$ & $\mathbf{2 0 0 7}$ & $\mathbf{2 0 0 8}$ & $\mathbf{2 0 0 9}$ & $\mathbf{2 0 1 0}$ \\
\hline Air Seychelles & 14 & 15 & 15 & 16 & 16 & 14 \\
Emirates & 6 & 4 & 4 & 4 & 4 & 7 \\
Qatar & 4 & 4 & 4 & 4 & 4 & 4 \\
Air Austral & 1 & 1 & 1 & 1 & 1 & 2 \\
Condor & 1 & 1 & 1 & 1 & 1 & 1 \\
Kenya Airways & 2 & 2 & 2 & 2 & 2 & 2 \\
Total Weekly Flights & $\mathbf{2 8}$ & $\mathbf{2 7}$ & $\mathbf{2 7}$ & $\mathbf{2 8}$ & $\mathbf{2 8}$ & $\mathbf{3 0}$
\end{tabular}

aln 2011, Ethiopian Airlines began flights to Seychelles. In April 2012, Air Seychelles began operating under Etihad. Emirates are now looking to increase weekly flights via Dubai.

Source: Seychelles Tourism Master Plan 2012-20: 35

Table A3.10 Production of key crops, 2007-2011

\begin{tabular}{cccc}
\hline Year & Copra (tonnes) & Cinnamon (tonnes) & Tea (tonnes) \\
\hline 2007 & 41 & 63 & 222 \\
2008 & 29 & 94 & 137 \\
2009 & 31 & 63 & 63 \\
2010 & 19 & 23 & 49 \\
2011 & 10 & 39 & 38 \\
\hline
\end{tabular}

Source: National Statistics Bureau, Seychelles

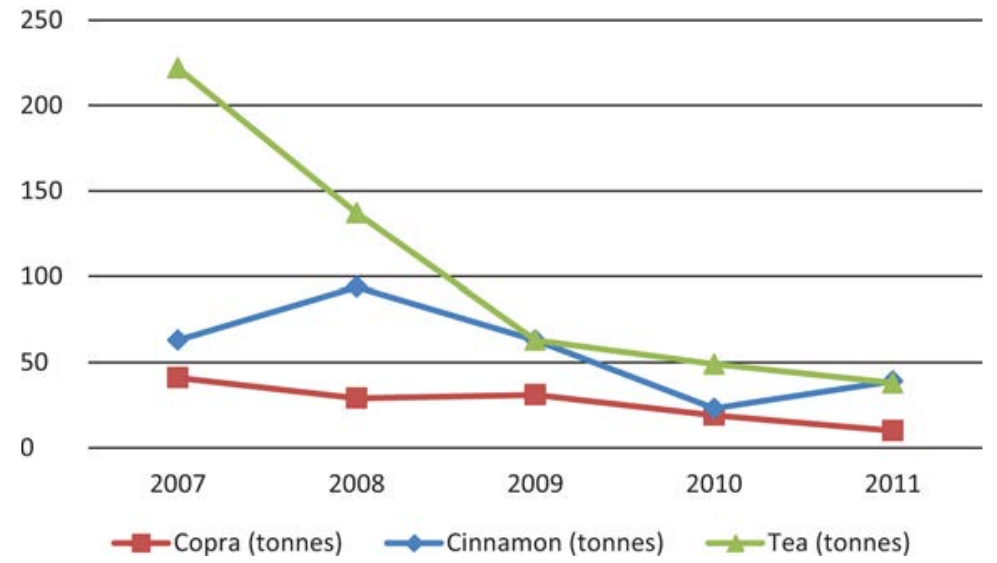


Table A3.11 Production of fish and animal feed, 2007-2011

\begin{tabular}{cccc}
\hline Year & Canned tuna (tonnes) & Fish catch (tonnes) & Animal feed (tonnes) \\
\hline 2007 & 31,569 & 4,211 & 13,881 \\
2008 & 28,907 & 4,800 & 13,053 \\
2009 & 30,824 & 3,364 & 10,015 \\
2010 & 30,338 & 2,597 & 9,053 \\
2011 & 30,152 & 2,875 & 4,088 \\
\hline
\end{tabular}

Source: National Statistics Bureau, Seychelles

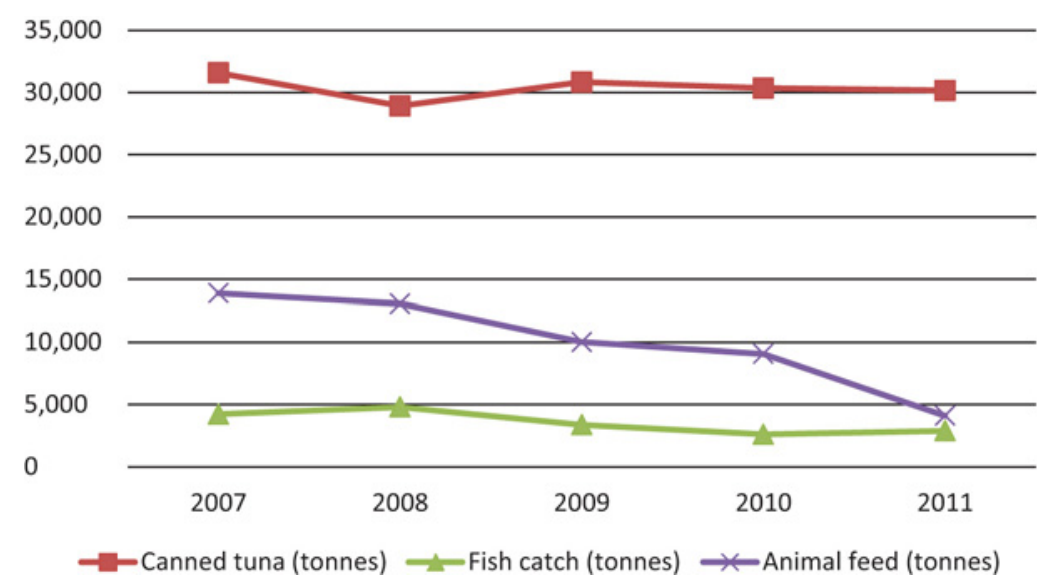

Table A3.12 Poultry production, 2007-2011

\begin{tabular}{cc}
\hline Year & Chickens (nos) \\
\hline 2007 & 833,320 \\
2008 & 768,115 \\
2009 & 606,697 \\
2010 & 550,685 \\
2011 & 622,303 \\
\hline
\end{tabular}

Source: National Statistics Bureau, Seychelles

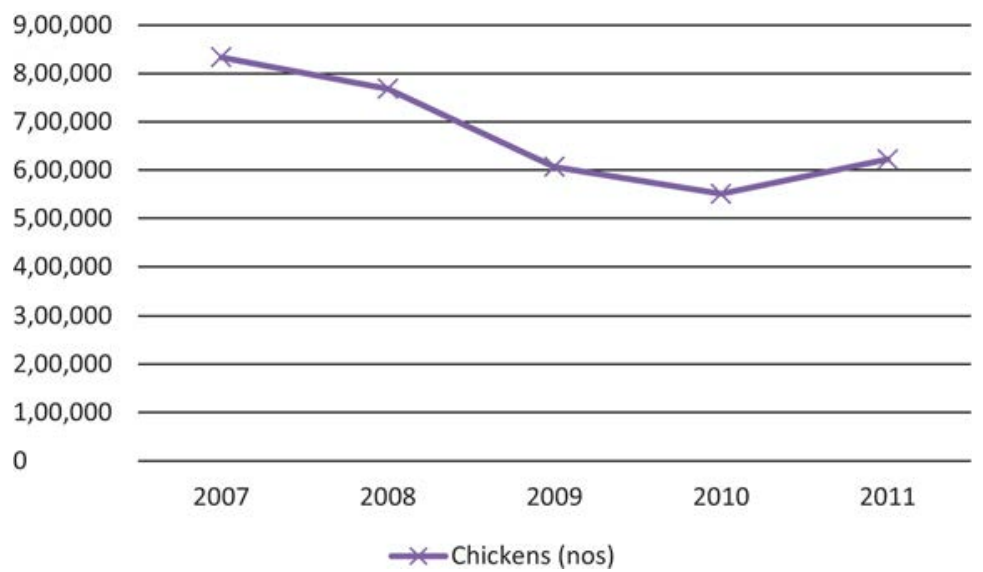


Table A3.13 Cattle production, 2007-2011

\begin{tabular}{cc}
\hline Year & Cattle (nos) \\
\hline 2007 & 75 \\
2008 & 54 \\
2009 & 35 \\
2010 & 34 \\
2011 & 15 \\
\hline
\end{tabular}

Source: National Statistics Bureau, Seychelles

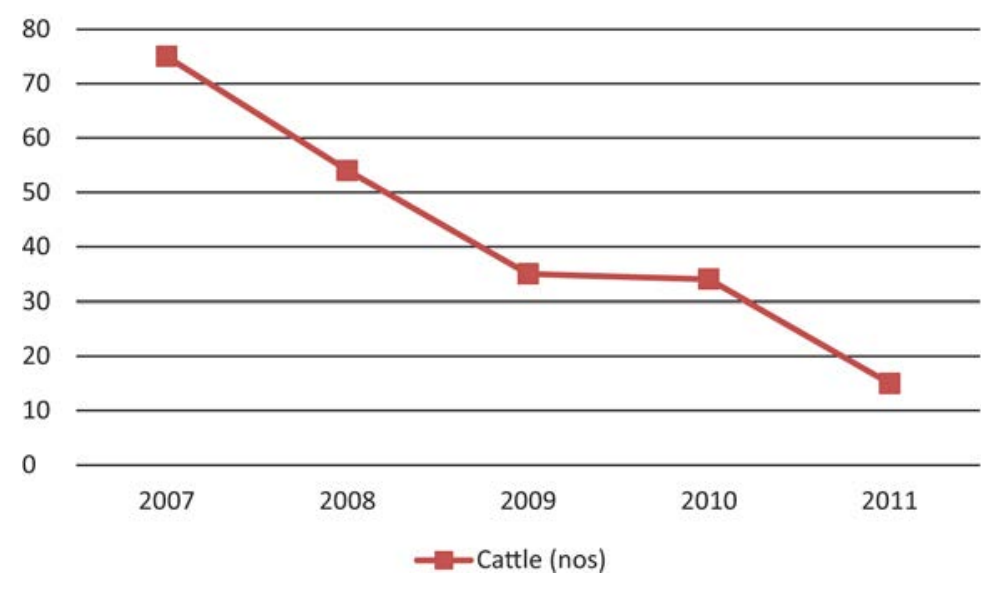


Table A3.14 Pig production, 2007-2011

\begin{tabular}{cc}
\hline Year & Pigs (nos) \\
\hline 2007 & 9,036 \\
2008 & 7,293 \\
2009 & 6,014 \\
2010 & 5,363 \\
2011 & 6,555 \\
\hline
\end{tabular}

Source: National Statistics Bureau, Seychelles

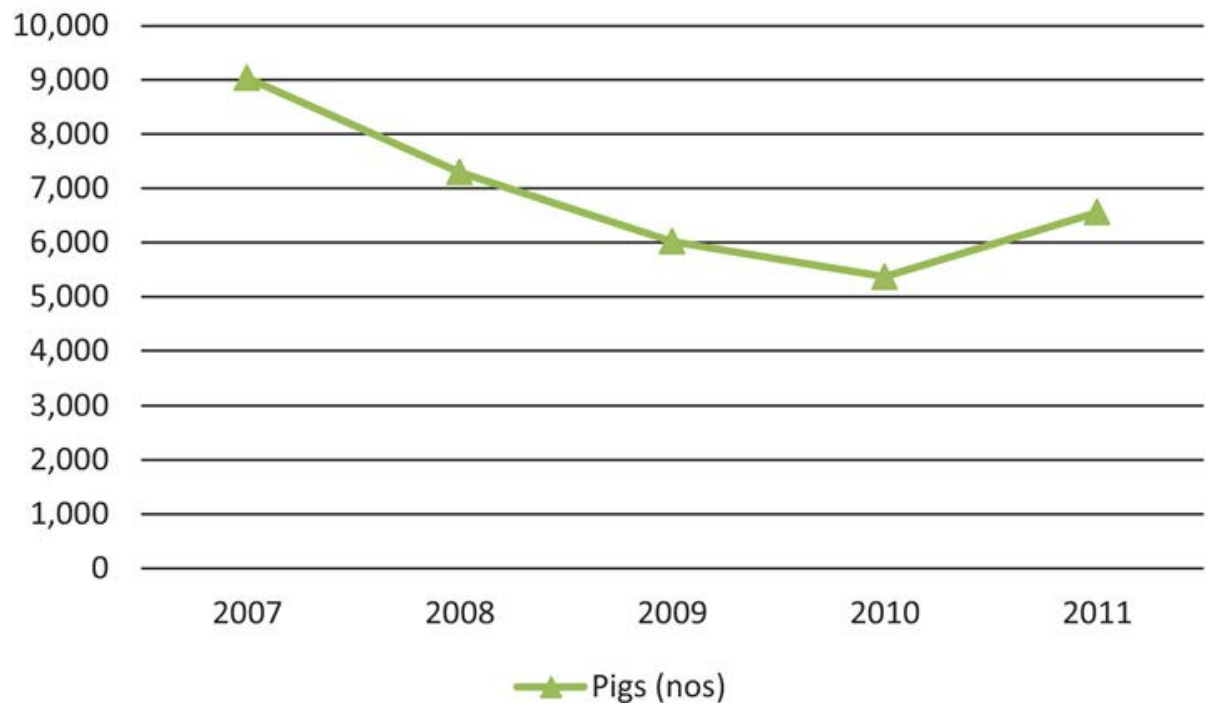

